\title{
Ĵigo: um editor de objetos de aprendizagem de segunda geração.
}

Marcelo Leandro Eichler* Gabriela Trindade Perry ${ }^{* *}$ José Claudio Del Pino*

Resumo: No Brasil e no Mundo, é notável a ampliação da produção de conteúdos digitais na forma de objetos de aprendizagem, porém ainda são raros os objetos de aprendizagem construídos tirando proveito das características excepcionais dessa mídia. De acordo com nossa experiência no desenvolvimento de conteúdos digitais, um dos pontos críticos é a parte tecnológica e de programação. Neste artigo, portanto, descrevemos uma ferramenta simples que pode ser utilizada na produção de objetos de aprendizagem de representações contextuais, visando à educação em ciências. Também buscamos apresentar alguns tópicos da didática das ciências, de pedagogia e de psicologia, como subsídios à informática educativa, com o intuito de mostrar como a utilização de objetos de aprendizagem, combinados com o debate escolar, constituem-se em uma importante estratégia para a educação em ciências.

Palavras-chave: objetos de aprendizagem, didática das ciências, florestamento.

Abstract: [Ĵigo: an second generation's learning objects editor] In worldwide, it's remarkable the large production of digital content like learning objects, but is still rare the learning objects design taking advantages of exceptional characteristics of this media. In accordance with our experience in the development of digital content, a critical point is the technology and programming. In this article, therefore, we present a simple tool that can be used in the production of contextual representation's learning objects for science education. Also we present some topics of science teaching, of pedagogy and psychology, as subsidies to educational informatics, with the goal to show how the use of learning objects, combined with the school debates, constitute an important strategy for science education.

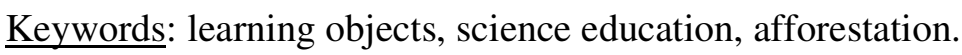

\footnotetext{
* Licenciado em Química e Doutor em Psicologia do Desenvolvimento pela UFRGS, é pesquisador da Área de Educação Química (AEQ) da UFRGS. Autor correspondente: exlerbr@yahoo.com.br ** Bacharel em Design, com habilitação em Programação Visual, pela ULBRA, é doutoranda em Informática Educativa pela UFRGS.

*** Licenciado em Química PUC-RS, Especialista em Ensino de Química UCS, Doutor em Química de Biomassa UFGRS, é professor do Instituto de Química e coordenador da AEQ-UFRGS.
} 


\section{Introdução}

No Brasil e no Mundo, é notável a ampliação da produção de conteúdos digitais na forma de objetos de aprendizagem, o surgimento de políticas públicas visando a ampliar essa produção e o aparecimento de repositórios para a distribuição desses conteúdos. Porém, em uma análise realizada no Brasil se mostra que "todos concordam com o grande potencial das novas tecnologias para revolucionar a educação, mas apesar das grandes expectativas sobre o potencial dessa nova ferramenta de aprendizagem, ainda são raros os objetos de aprendizagem construídos tirando proveito das características excepcionais dessa mídia" (Nascimento, 2007, pp. 135-136).

A expectativa sobre esses recursos educacionais digitais, na forma de simulações e atividades interativas, é a de que a aprendizagem se torne mais efetiva e mais profunda que a obtida pelos meios tradicionais. Entretanto, essa realização não é a regra. Em uma avaliação dos objetos de aprendizagem disponíveis através do atual programa público brasileiro (RIVED), constatou-se que "[os] autores e [as] equipes de produção muitas vezes deixam-se influenciar mais pelo potencial lúdico que pelo potencial de aprendizagem de seus produtos, resultando em atividades que entretêm o aluno, mas com as quais ele não aprende. Outras vezes criam situações monótonas e que não aproveitam o potencial de programação do computador para obter níveis altos de interatividade, visualização e manipulação" (Nascimento, 2007, p. 137).

Uma experiência internacional interessante e importante de ser citada é da Oceania. Atualmente, na Austrália, distribui-se ao meio escolar cerca de 6200 itens agrupados em diversos eixos temáticos (http://econtent.thelearningfederation.edu.au). Por exemplo, em relação ao ensino de ciências, os objetos de aprendizagem são agrupados sob os temas transversais: i) Planeta Terra e além; ii) energia e mudança; iii) vida e subsistência; iv) materiais naturais e v) processos e trabalho científico.

Uma avaliação desse programa pode ser encontrada em Schibechi e colaboradores (2008). A avaliação realizada por esses autores indicou que os objetos de aprendizagem possuem uma variedade de benefícios aos estudantes. Em geral, os estudantes apreciaram a novidade e acharam os objetos de aprendizagem motivadores. Os professores, por sua vez, declararam o aumento dos níveis de concentração, de entusiasmo e de aprendizagem dos alunos quando eles utilizaram os objetos de aprendizagem. Isso porque os estudantes foram motivados a utilizar os objetos de aprendizagem através de desafios, que permitiam que eles explorassem e tivessem controle sobre suas ações, assim entusiasmavam a colaboração entre colegas. Nesse sentido, os objetos de aprendizagem levaram os estudantes resistentes às abordagens tradicionais de ensino e com baixos desempenhos acadêmicos a se engajarem em tarefas escolares e de aprendizagem. Nesses casos, observou-se que estudantes considerados problemáticos participaram ativamente das aulas. Também estudantes com pouco ou insuficiente conhecimento de leitura e escrita se engajaram fortemente às atividades, pois os objetos de aprendizagem conferem acesso e interação aos conceitos e conhecimentos por meios mais diversos que os textuais.

Nessa mesma avaliação, do ponto de vista dos projetistas e produtores dos objetos de aprendizagem, evidenciou-se que os conteúdos digitais podem ser aperfeiçoados de várias formas, por exemplo, através da melhor utilização de animações e de elementos visuais e sonoros, reduzindo o tamanho dos textos, indicando instruções de utilização, fornecendo caminhos de navegação e considerando a utilização de estratégias de exploração, resolução de problemas e de jogo.

Por isso que se reitera a conhecida importância da "formação de uma equipe multidisciplinar na qual alunos e professores especialistas em áreas de conhecimentos trabalhem colaborativamente com pedagogos, professores de informática, 
programadores e web designers" (Nascimento, 2007, p. 136). Porém, de acordo com nossa experiência no desenvolvimento de conteúdos digitais, um dos pontos críticos é justamente a parte tecnológica e de programação (Eichler e Del Pino, 2001).

Neste artigo, portanto, buscamos apresentar uma ferramenta simples que pode ser utilizada na produção de objetos de aprendizagem para a educação em ciências, utilizando estratégias de solução de papéis e de debates escolares por representação de papéis.

Alguns tópicos da didática das ciências, como subsídio à informática educativa.

A escolha dos temas e o planejamento das atividades a serem apresentados nos objetos de aprendizagem podem seguir orientações das abordagens de ciência, tecnologia e sociedade (CTS) para a educação em ciências. Essa abordagem, conforme Gilbert (1992), possui algumas relevantes contribuições na educação científica: i) o nível de abstração pode ser reduzido; ii) o conhecimento pode ser reorganizado ('repackaged', no original), pelos professores; iii) o conhecimento pode ser reconstruído, pelos alunos; e iv) o conhecimento pode ser contextualizado.

De acordo com Ankiewicz e colaboradores (2006), os primeiros momentos dos estudos CTS foram úteis para ampliar o reconhecimento dos efeitos da poluição ambiental e dos movimentos de consumidores dos anos 1960, cujo ímpeto resultou em preocupações em relação à energia nuclear e o impacto social das mudanças tecnológicas, como a automação, a crise energética, o desenvolvimento da engenharia genética e temas similares.

A contextualização é um princípio-chave dessa abordagem. Por exemplo, Solbes e Vilches (1997) realizaram uma investigação sobre a utilização de temáticas de CTS em aulas de física e de química em conjunto com um modelo didático de ciência como pesquisa e concluíram que: (1) os estudantes que participaram dessa inovação curricular possuem uma idéia acerca da ciência mais realística e contextualizada, mostrando diferenças significativas de estudantes que seguem o ensino chamado de tradicional; e (2) é possível ampliar a participação dos estudantes e aumentar seu interesse em estudar física e química negociando com eles os assuntos que serão abordados em aula.

$\mathrm{Na}$ escolha das temáticas, Haney e colaboradores (2007) indicam que professores do ensino médio podem trabalhar, nas aulas de disciplinas científicas, com temas interdisciplinares baseados em problemas relevantes e locais, envolvendo assuntos de saúde pública ou ocupacional. Exemplos dessa temática envolvem: a qualidade do ar nos prédios escolares, as práticas agrícolas em larga escala, a utilização de agrotóxicos, o impacto da urbanização na saúde humana e a segurança de produtos sanitários e desinfetantes domésticos, entre outros. Porém, através de uma pesquisa no contexto da formação de professores, Gil-Pérez e colaboradores (2003) evidenciaram que os problemas mais mencionados, em função de sua utilização na abordagem de CTS, são a poluição ambiental, a urbanização desordenada e o esgotamento de recursos naturais, que estão estreitamente relacionados e provocam a degradação da vida no planeta.

Segundo Dillon (1993), as tecnologias relacionadas ao crescimento econômico e à criação de riqueza estão associadas com os principais impactos ambientais, através das demandas que elas têm por energia e por outras matérias-primas. Dessa forma, a tensão entre crescimento econômico e as externalidades ${ }^{\dagger}$ ambientais podem ser exploradas pela

\footnotetext{
$\dagger$ Externalidades, também chamadas economias (ou deseconomias) externas, são efeitos positivos ou negativos - em termos de custos ou de benefícios - gerados pelas atividades de produção ou consumo exercidas por um agente econômico e que atingem os demais agentes, sem que esses tenham
} 
educação em ciências através de diversos temas, por exemplo, a acidificação da chuva, o desflorestamento e o aquecimento global.

Nesse sentido, Hodson (2003) argumenta que para os problemas sociais e ambientais contemporâneos serem resolvidos é necessária uma geração de cidadãos formados política e cientificamente que não se contentem com o papel da "crítica de escrivaninha". O autor sugere um currículo focalizado em assuntos controversos e politizado em sete diferentes áreas (saúde humana; alimentação e agricultura; terra, água e recursos minerais; recursos energéticos e consumo; indústria; tecnologias da informação; e responsabilidade técnica e sociopolítica) e propõe quatro níveis de sofisticação:

1) Apreciar o impacto social das transformações científicas e tecnológicas, reconhecendo que a ciência e a tecnologia são, em alguma extensão, culturalmente determinadas;

2) Reconhecer que as decisões acerca do desenvolvimento científico e tecnológico são tomadas em busca de interesses particulares e que os benefícios e lucros de uns crescem ao custo e prejuízo de outros, reconhecendo que o desenvolvimento científico e tecnológico está inseparavelmente ligado à distribuição de riqueza e poder.

3) Desenvolver os próprios pontos de vista e estabelecê-los em relação aos valores subjacentes.

4) Preparação para a ação sociopolítica.

Dessa forma, ele postula que a educação científica para a ação sociopolítica é, inescapavelmente, um exercício de aclaração e mudança de valores, isso porque os valores não podem e não devem ser impostos aos estudantes, mas sua adoção pode ser encorajada.

O autor reconhece que junto ao movimento CTS, amplia-se o reconhecimento da necessidade de olhar as práticas sociais, políticas, econômicas e éticas que cercam as práticas sociais. Assim, a ciência perdeu muito da inocência e da pureza sugerida pelos criadores das reformas curriculares da década de 1960 (projetos Nuffield e Chem Study, por exemplo). Uma sucessão de tragédias humanas e ambientais tem evidenciado, algumas vezes, o papel de vilão da ciência. As mudanças distributivas sociais e as preocupações profundamente éticas e morais surgidas com as inovações científicas e tecnológicas tem levado a ciência a ser vista por muitos como uma ameaça aos modos de vida confortáveis e familiares. A crescente comercialização, industrialização e militarização das ciências têm mostrado, de uma vez por todas, que a ciência não é desinteressada e livre de valores. Portanto, a ciência necessária para a solução de problemas cotidianos e contemporâneos é muito diferente daquele que é apresentada através do currículo escolar.

Do ponto de vista da ecologia política, por exemplo, Martínez Alier (2007), interroga-se "sobre quem possui o poder político para simplificar a complexidade e sacrificar certos interesses e valores sociais impondo um único discurso de valoração a despeito dos demais, como tem ocorrido com o discurso econômico" (p. 14). Conforme esse autor, a distribuição precede as decisões de produção. Assim, considerando a distribuição ecológica, pode-se argumentar que não será tomada nenhuma decisão sobre a produção enquanto não existir um acordo ou norma habitual sobre como os recursos

oportunidade de impedi-los ou a obrigação de pagá-los. Portanto, externalidades referem-se ao impacto de uma decisão sobre aqueles que não participaram dessa decisão. A externalidade pode ser negativa, quando gera custos para os demais agentes - a exemplo, de uma fábrica que polui o ar, afectando a comunidade próxima. Pode ser positiva, quando os demais agentes, involuntariamente, se beneficiam, a exemplo dos investimentos governamentais em infra-estrutura e equipamentos públicos (Wikipédia). 
naturais serão apropriados ou como serão destinados seus resíduos. Por exemplo, "a decisão de produzir energia elétrica a partir do carvão requer uma decisão prévia sobre a destinação dos dejetos da mineração, sobre o dióxido de enxofre, os óxidos de nitrogênio e o dióxido de carbono em distintas escalas geográficas. Quem desfruta do direito de propriedade sobre esses lugares? Em termos econômicos, se as externalidades podem permanecer como tais - isto é fora da contabilidade dos resultados e do balanço da empresa -, as decisões seriam diferentes caso tais passivos ambientais fossem incorporados na sua conta (inserindo algum valor econômico). (...) Por exemplo, se uma fábrica de celulose no Brasil pode plantar eucaliptos ignorando a compensação pela perda de fertilidade e pode lançar os efluentes exercendo de fato direitos de propriedade sobre o rio ou o mar, suas decisões de produção são diferentes das que existiriam na hipótese de se ver obrigada a pagar por essas externalidades ou caso tivesse que se confrontar com normas legais mais estritas, sendo essas levadas a efeito à risca" (grifos nossos; pp. 51-52).

Por fim, fechando a discussão da temática que utilizamos como exemplo no lançamento de nosso editor de objetos de aprendizagem, Vacik e colaboradores (2006), indicam que os estudantes devem aprender a inter-relação dos conhecimentos técnico, ecológico e sócio-econômico subjacente ao gerenciamento dos recursos naturais e à silvicultura. Por isso, deve-se considerar que a tomada de decisão no planejamento da silvicultura envolve aspectos ecológicos, econômicos e sociais, em níveis locais, nacionais e globais. Portanto, entendemos que tal recomendação pode ser seguida através de estratégias didáticas que utilizem o estudo de caso, visando à tomada de decisão, em cenários apresentados em objetos de aprendizagem.

Alguns tópicos de pedagogia e de psicologia, como subsídio à informática educativa.

Seguindo o que foi abordado na secção anterior, é possível constatar, conforme Gilbert (2008), que os aspectos científicos e tecnológicos que são contemporâneos são quase sempre controversos. Então, dada a possibilidade da existência de visões de mundo tendenciosas, a educação acerca de temas controvertidos deve estar baseada na explicitação dos pontos-de-vista e do quadro moral que os subscreve. Nesse sentido, conforme apresentamos a seguir, entendemos que a utilização de objetos de aprendizagem de representações contextuais, combinados com o debate escolar, constitui-se em uma importante estratégia para a educação em ciências.

De acordo com Marjanovic (2007), a adoção dos princípios teóricos de um projeto de aprendizagem assegura que todas as atividades educacionais sejam guiadas pela pedagogia e não apenas pela utilização indiferenciada dos recursos educacionais ou tecnológicos disponíveis. Um exemplo recorrente de projetos de aprendizagem é o processo de resolução de problemas, que é amplamente utilizado em diversas disciplinas. Embora o atual desenvolvimento da aprendizagem baseada em problemas possa variar, em orientação teórica ou metodológica, esse projeto de aprendizagem incorpora as seguintes atividades: identificação das alternativas, análise das alternativas identificadas e seleção de uma possível solução para o problema, podendo ser seguindo pelo planejamento de uma estratégia de ação visando a tal solução.

Acerca do uso de cenários, Hinostroza e Mellar (2001) entendem que a utilização de histórias para apoiar a apresentação do conteúdo de uma lição é uma estratégia didática comum, descria muitas vezes como um modo de "familiarizar" a criança ou o jovem com o conteúdo curricular e estabelecer um conhecimento contextualizado. Assim, pode-se usar essa estratégia em software e projetá-los de tal forma que os usuários possam imergir na história e tornar-se protagonista das atividades, sendo possível manipular elementos presentes nos diferentes cenários da 
interface e interagindo com o conteúdo dos exercícios ou desafios que guiam o estudante através do software.

Segundo Martínez Pérez e colaboradores (2007), acerca do debate de argumentos sobre problemáticas ambientais, como a identificação das fontes e causas de contaminação atmosférica e seus prejuízos sociais, é possível identificar os atores sociais que participam das controvérsias, tais como o ator social, o ator industrial, o ator científico, o ator político, o ator educativo e o ator moderador.

Do ponto de vista da psicologia da aprendizagem, Parrat-Dayan (2007) sugere que a discussão e o diálogo na escola se tornam ferramentas essenciais para a construção do pensamento e da socialização. A discussão é uma importante ferramenta que incita a criança ou o jovem à criatividade, ao espírito crítico, à confrontação de pontos de vista diferentes. Assim, o conflito cognitivo, que provoca a diversidade de pontos de vista entre pares, permite que os alunos se questionem, duvidem, problematizem a realidade, proponham soluções, critiquem as alternativas sugeridas e identifiquem os critérios para achar a solução mais coerente. Portanto, a discussão e o debate são elementos imprescindíveis de uma pedagogia que pretende desenvolver o pensamento das crianças, a cidadania e a democracia, ou seja, o direito de expressar idéias num espaço coletivo e público, onde se admite o pluralismo.

Portanto, conforme Oulton e colaboradores (2004), o ensino acerca desses temas controversos deve enfatizar que: i) os grupos em sociedade possuem diferentes visões acerca do assunto; ii) os grupos baseiam suas informações em diferentes conjuntos de informações ou interpretam as mesmas informações de modos diversos; iii) as diferentes interpretações podem ocorrer devido às diferentes formas de "ver" o mudo, ou seja, devido às diversas cosmovisões; iv) as diversas comovisões podem ocorrer por que os indivíduos aderem a diferentes sistemas de valor; v) assuntos controversos não podem ser sempre circunscritos à razão, à lógica e ao experimento; e vi) assuntos controversos podem ser resolvidos tornando mais informações disponíveis.

Algumas considerações sobre as características desejáveis em objetos de aprendizagem.

Conforme Wiley (2000), a idéia fundamental subjacente aos objetos de aprendizagem é que seus projetistas instrucionais podem construir pequenos componentes de instrução (relativo ao tamanho de um curso inteiro) que podem ser reusados diversas vezes em diferentes contextos educacionais. Mas é preciso notar, como sugerem Littlejohn e colaboradores (2008), que a manipulação e a interação são aspectos-chave para a afetiva aprendizagem, pois é através da imersão digital que os usuários constroem seus próprios entendimentos acerca do que está representado, extraindo dali os propósitos e valores educacionais.

Assim, segundo Parrish (2004), a utilização de objetos de aprendizagem apresenta um importante papel de suporte em estratégias ativas de aprendizagem, como aquelas baseadas em estudos de caso, ou em resolução de problemas, ou em formas colaborativas de debates argumentativos. Nessas estratégias, os objetos de aprendizagem promovem estímulo e suporte para a prática de tarefas complexas, não apresentando apenas informações ou guiando um percurso previamente determinado, como na coleção de lições estáticas por algoritmos pré-definidos.

Nesse sentido, Allert e colaboradores (2004) delinearam um tipo de recurso educacional projetado para a efetiva promoção e geração da aprendizagem, chamando-o de objeto de aprendizagem de segunda ordem. Conforme esses autores, os objetos de aprendizagem de primeira ordem são recursos criados ou redesenhados visando um objetivo de aprendizagem específico. Assim, apresentam uma informação que deve ser adquirida, redescoberta ou reconstruída. Exemplos desse tipo de objeto de 
aprendizagem são os livros-texto, filmes educacionais e outras formas simples de apresentação de informação. Por sua vez, os objetos de aprendizagem de segunda ordem são recursos que contém ou provêem estratégias de aprendizagem como, por exemplo, a resolução de problemas, a tomada de decisão ou o planejamento estratégico.

De acordo com Churchill (2007), os objetos de aprendizagem são projetados para suprir sua utilização em diferentes contextos educacionais. Dessa forma, propõe uma classificação para os objetos de aprendizagem, que conteria objetos de apresentação, objetos de prática, objetos de modelos conceituais, objetos de informação e objetos de representação contextual. Segundo essa classificação, a idéia subjacente aos objetos de representação contextual é permitir aos estudantes a exploração de algum cenário realístico, onde ele possa coletar dados, normalmente com o propósito de resolução de problemas ou investigação. Os exemplos trazidos pelo autor envolvem temas como a atividade vulcânica, as condições climáticas, os poluentes atmosféricos, as formas de vida em grandes profundidades oceânicas e o relato da opinião das pessoas acerca desses assuntos ou de outros.

Geralmente, utiliza-se a representação contextual de algum lugar imaginário ou inacessível aos estudantes, devido a, por exemplo, sua distância, no tempo ou no espaço, ou ao perigo envolvido em seu acesso e exploração, ou a escala muito pequena ou muito grande para a coleta de dados, ou a necessidade de instrumentos sofisticados para tal, entre outros. Nesse sentido, o autor descreve uma representação contextual acerca da qualidade da água. No objeto de aprendizagem produzido, os alunos podem coletar dados acerca dos fatores que afetam a qualidade da água em um lago imaginário, apresentado como cenário. Tais dados podem ser usados em uma atividade de resolução de problemas que sugere aos alunos agirem como ambientalistas, investigando a situação e propondo uma solução para o problema na forma de um relatório para uma agência de proteção ambiental.

Ou seja, essa idéia é muito similar aquilo que vimos produzindo (Eichler e Del Pino, 2006), inspirados no trabalho de Whisnant (1984 e 1992). Porém, a questão tecnológica e a programação continuam sendo pontos sensíveis à produção dos objetos de aprendizagem.

É nesse ponto que relevante a proposição de Morales e colaboradores (2005), que introduzem o conceito de um editor de objetos de aprendizagem (Generative Learning Objects - GLO, no original), baseado na separação entre o projeto de aprendizagem e a instanciação da fachada do objeto de aprendizagem. O conceito-chave de um editor de objetos de aprendizagem é separar a estrutura profunda (o projeto pedagógico ou projeto de aprendizagem) da estrutura de superfície (a fachada ou a apresentação) dos objetos de aprendizagem. A fachada do objeto de aprendizagem é vista com uma realização particular de um projeto de aprendizagem subjacente. Isso apresenta muitas vantagens. Centra-se a maior atenção sobre o que é mais importante, a qualidade do projeto de aprendizagem que será apoiada pelo objeto digital, como abordamos nas secções anteriores. Uma vez que a fachada do objeto é gerada em uma série de passos, muitas variantes podem ser produzidas com o mesmo projeto. Além disso, uma vez que os objetos de aprendizagem são divididos em componentes básicos e estruturais, torna-se fácil identificar e modificar componentes individuais. Assim, nos editores de objetos de aprendizagem, utiliza-se um modelo de reuso para a estrutura de superfície, o que está próximo da engenharia de software orientada a objeto. Isso produz diversas vantagens, entre elas: i) focaliza a atenção na qualidade do projeto de aprendizagem, que é o cerne do objeto de aprendizagem; e ii) apresenta o fundamento para uma marcante ampliação da produção de objetos de aprendizagem. 
Segundo esses autores, a criação de um editor de objetos de aprendizagem é dividida em duas amplas partes, a construção de um gabarito para os objetos de aprendizagem (Learning Object Template, no original) e a posterior adição dos conteúdos específicos. Esse gabarito, ou modelo de objeto de aprendizagem, contém a estrutura profunda ou o projeto de aprendizagem. Uma vez criado o gabarito, tutores (professores ou alunos, por exemplo) podem adicionar diferentes conteúdos específicos, produzindo diversos objetos de aprendizagem, adaptados aos propósitos de suas áreas de conhecimento, que depois de revisados e analisados por avaliadores podem ser publicados e distribuídos pela Internet. Nesse sentido, os autores apresentam um editor de objetos de aprendizagem cujo conceito de método estatístico é o seu cerne. A partir desse editor, por exemplo, foram desenvolvidas atividades nas áreas de psicologia, criminologia, política, enfermagem e saúde pública.

Porém, como indicam, é preciso e urgente criar ferramentas amigáveis para suportar esse processo de criação dos objetos de aprendizagem. A ferramenta que apresentamos na próxima secção, Jigigo, é um editor de objetos de aprendizagem que contém um gabarito para a produção de representações contextuais.

$\underline{\text { Um editor de objetos de aprendizagem: Jigo }}$

O objetivo de Ĵigo é propiciar um ambiente de criação e de utilização de objetos de aprendizagem que permitam a professores e alunos buscar a apresentação e a discussão de temas relacionados ao meio ambiente. Em Jigo, a estrutura de fachada, de apresentação, pode ser editada. Como estrutura profunda nós estabelecemos a utilização de dois projetos pedagógicos ou de aprendizagem, apoiados em cenários: resolução de problemas e debate escolar. Portanto, Jigo é um gabarito, uma template para esses tipos de atividade pedagógica. Para o nome desse gabarito e do seu editor foi adotado o termo ‘jigo', do esperanto, que sugere idéias de jogo, quebra-cabeça, jogo de paciência e puzzle, por exemplo.

A ferramenta está disponível em: http://www.iq.ufrgs.br/aeq/jigo/, e foi desenvolvida utilizando a tecnologia Flex, que é um framework de código aberto para a criação de aplicativos ricos de internet $\left(\right.$ RIAs $\left.^{\ddagger}\right)$, altamente interativos. Dentro dele, pode-se usar MXML (uma linguagem declarativa baseada em XML) para especificar comportamentos e a interface com o usuário, e Actionscript 3.0, uma linguagem de programação orientada a objetos e executada em uma máquina virtual chamada AVM Actionscript Virtual Machine - que está disponível no plug-in Flash Player.

A seguir, descrevemos as principais etapas de edição da estrutura de apresentação dos objetos de aprendizagem. Nesse sentido, usamos como exemplo a temática do florestamento de eucaliptos (Hasse, 2006; Vaz 2008a, 2008b e 2008c).

No editor de objetos de aprendizagem, após o cadastramento do usuário, aparece uma tela semelhante à Figura 1, onde são inseridas as informações (título, assunto, descrição, palavras-chave e escolha da paisagem) que descrevem o objeto de aprendizagem planejado.

$\mathrm{Na}$ parte superior do editor há duas regiões que indicam as ações comuns (maximizar ou minimizar - a janela de edição - salvar, sair ou publicar) e ações de edição e criação, que é composta de cinco etapas: i) descrição da atividade; ii) áreas e dados; iii) personagens e mídias; iv) questões da atividade; e v) FAQ da atividade.

‡ RIA - Rich Internet Application - são aplicações web que se parecem com aplicações que rodam no desktop. O termo foi criado pela Adobe em 2002, para definir uma categoria de aplicativos que oferecem uma experiência superior. 
Na primeira etapa, de descrição do objeto de aprendizagem, o usuário (por exemplo, um professor) deve escolher entre as ilustrações oferecidas que possibilitam a ambientação e a contextualização das atividades que se propõe a desenvolver. Atualmente são oferecidas 5 paisagens de regiões urbana, rural, litorânea e montanhosa.

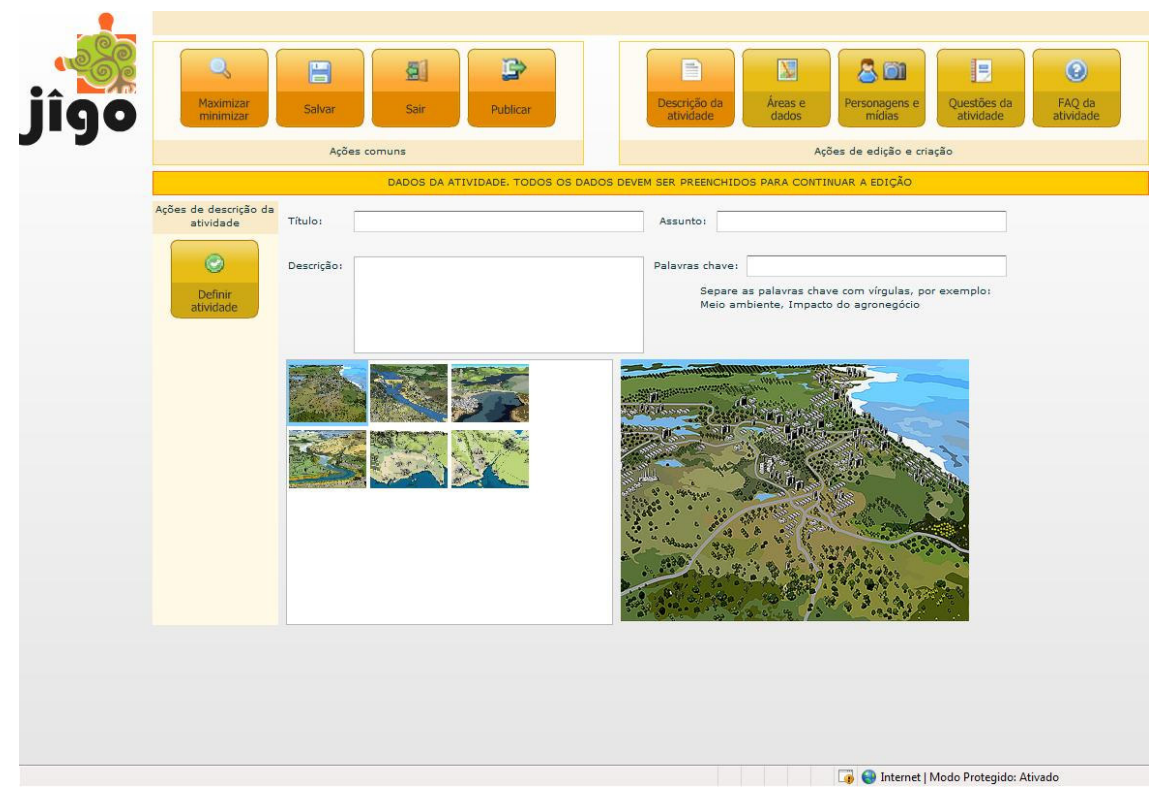

Figura 1 - Tela inicial de edição dos objetos de aprendizagem.

O primeiro objeto de aprendizagem produzido através de Ĵigo tem como assunto a indústria de papel e celulose e os impactos ambientais e sociais da silvicultura. Então, nessa primeira etapa, indicou-se que: "De acordo com os debates acerca do desenvolvimento econômico sustentável, o eucalipto tornou-se simultaneamente alvo de ataques raivosos dos defensores do equilíbrio ambiental e objeto de defesa apaixonada dos adeptos da silvicultura comercial. Por coincidência, a polêmica sobre o avanço do eucalipto em bioma predominantemente campestre (como os pampas, gaúcho e platino) ocorre exatamente no mesmo momento em que se discute a validade do desmatamento da Amazônia para o plantio de lavouras de grãos (como a soja, por exemplo). Nesse sentido, o eucalipto vem assumindo um lugar como substituto de madeiras tropicais cuja exploração é cada vez mais difícil por razões geográficas e ambientais. Assim, neste objeto de aprendizagem, utiliza-se um cenário de florestamento de eucaliptos como representação contextual visando ao debate escolar (por exemplo, através de jogo de representação de papéis) e a reflexão em torno da pertinência de determinadas escolhas econômicas e seus impactos nas comunidades terrestres. No objeto de aprendizagem podem ser lidos depoimentos de personagens que apresentam as diversas versões acerca do assunto, podem ser coletadas informações sobre a qualidade do solo e podem ser vistos vídeos e imagens que ilustram a temática. Caso necessário, pode ser acessada uma aba de ajuda, elaborada através de perguntas e respostas. Além disso, há um questionário a ser respondido após a utilização do objeto de aprendizagem".

$\mathrm{Na}$ segunda etapa, são atribuídas tabelas de dados relacionados às diferentes áreas da paisagem. Em um outro exemplo, se um professor pretende criar um cenário visando ao debate sobre os agrotóxicos no meio ambiente, ele pode escolher uma paisagem de uma microrregião de transição entre o rural e o urbano, alimentar o banco de dados com as unidades de concentração de agrotóxicos (p.ex., organofosforados ou carbamatos) e estipular diferentes concentrações para diversas áreas da paisagem. Nesse 
exemplo, durante a utilização do objeto de aprendizagem, os alunos, poderiam vir a pesquisar a concentração de agrotóxicos em diferentes locais da paisagem. Esse tipo de atividade é similar aquela que desenvolvemos no software Carbópolis (www.iq.ufrgs.br/aeq/carbop). No caso do objeto de aprendizagem sobre o florestamento de eucaliptos, inserimos dados sobre o índice de acidez $(\mathrm{pH})$ do solo e níveis de concentração de íons salinos $\left(\mathrm{Na}^{+}, \mathrm{K}^{+}\right.$e $\mathrm{SO}_{4}{ }^{2-}$, entre outros) em diferentes regiões do cenário, no mapa ilustrativo.

$\mathrm{Na}$ terceira etapa, são inseridas imagens e personagens que servem para ilustrar os diferentes pontos-de-vista de um assunto. Nessa etapa, o usuário poderá editar um determinado número de personagens (escolhendo gênero, etnia e vestimentas, por exemplo) que serão inseridos em diferentes locais da paisagem, conforme Figura 2. Nesse tipo de atividade, os usuários devem indicar os depoimentos desses personagens em relação à temática que esteja em discussão. Por exemplo, se um professor pretende criar um cenário visando ao debate sobre os alimentos geneticamente modificados, pode escolher uma paisagem de uma macrorregião, depois solicitar que os alunos montem os personagens e que indique os depoimentos que os personagens fariam sobre o tema em debate. A montagem inicial desse cenário serve de suporte para os jogos de representação de papéis, conforme atividade similar que desenvolvemos no software Cidade do Átomo (www.iq.ufrgs.br/aeq/cidatom). No caso do objeto de aprendizagem sobre o florestamento de eucaliptos, utilizamos diversas opiniões apresentadas em Hasse (2006), informações econômicas e ambientais relatadas por Hilgemberg e Bacha (2003) e imagens e argumentos reportados por Vaz (2008a, 2008b e 2008c).

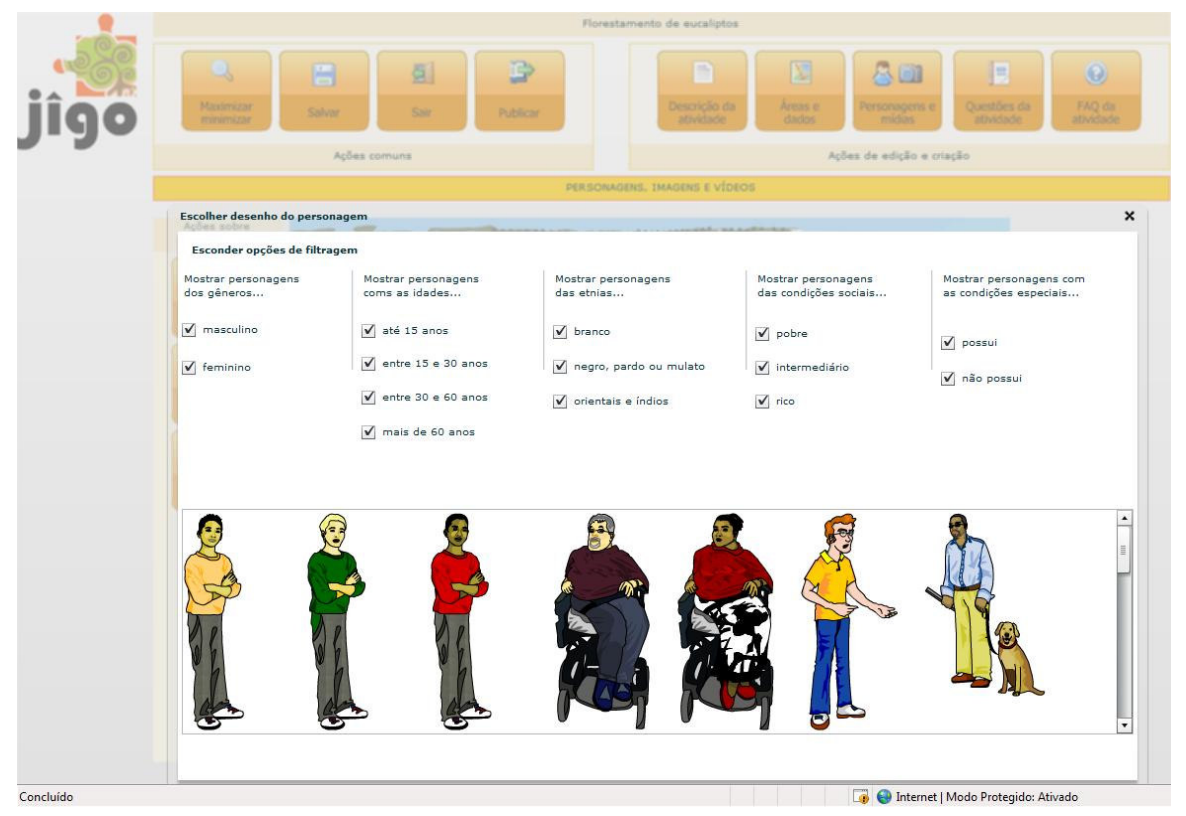

Figura 2 - Tela de escolha dos personagens para inserção nos objetos de aprendizagem.

A terceira e quarta etapas consistem na elaboração dos textos de apóio a utilização do objeto de aprendizagem, seja na forma de questionários que indiquem uma conclusão para as atividades, seja um conjunto de perguntas e respostas que serve para dirimir as dúvidas dos usuários acerca da utilização do objeto de aprendizagem planejado ou do conteúdo apresentado. 
Por fim, ao encerrar este artigo, é preciso dizer que, posteriormente, conforme realizamos com Carbópolis (Eichler e Del Pino, 2006), após uma ampla divulgação junto aos sistemas escolares, pretendemos verificar como esse tipo de material didático é efetivamente utilizado em contextos escolares, principalmente em relação às estratégias didáticas utilizadas pelos(as) professores(as).

Agradecimento:

À FINEP, pelo financiamento concedido que permitiu a elaboração do editor de objetos de aprendizagem.

Referências bibliográficas:

ALLERT, H.; RICHTER, C. \& NEJDL, W. Lifelong learning and second-order learning objects. British Journal of Educational Technology, 35, 701-715, 2004.

ANKIEWICZ, P.; DE SWARDT, E. \& DE VRIES, M. Some implications of the philosophy of technology for Science, Technology and Society (STS) studies. International Journal of Technology and Design Education, 16, 117-141, 2006.

CHURCHILL, D. Towards a useful classification of learning objects. Educational Technology Research and Development, 55, 479-497, 2007.

DILLON, P.J. Technological education and the environment. International Journal of Science Education, 15 (5), 575-589, 1993.

EICHLER, M.L. \& DEL PINO, J.C. Algumas reflexões sobre o desenvolvimento de um projeto de informática educativa, em época de ajuste fiscal. Tecnologia Educacional, 30 (154), 57-69, 2001.

EICHLER, M.L. \& DEL PINO, J.C. Ambientes virtuais de aprendizagem: desenvolvimento e avaliação de um projeto em educação ambiental. Porto Alegre: Editora da UFRGS, 2006.

GIL-PÉREZ, D.; VILCHES, A.; EDWARDS, M.; PRAIA, J.F.; VALDÉS, P.; VITAL, M.L.; TRICÁRIO, H.; \& RUEDA, C. A educação científica e a situação do mundo: um programa de atividades dirigido a professores. Ciência \& Educação, 9 (1), 123-146, 2003.

GILBERT, J.K. The interface between science education and technology education. International Journal of Science Education, 14 (5), 563-578, 1992.

GILBERT, J.K. Science communication: towards a proper emphasis on the social aspects of Science and Technology. Alexandria - Revista de Educação em Ciência e Tecnologia, 1 (1), 3-25, 2008.

HANEY, J.J.; WANG, F.; KEIL, C.; \& ZOFFEL, J. Enhancing teachers' beliefs and practices through problem-based learning focused on pertinent issues of Environmental Health Science. The Journal of Environmental Education, 38 (4), 25-33, 2007.

HASSE, G. Eucalipto: histórias de um imigrante vegetal. Porto Alegre: Já Editores, 2006.

HILGEMBERG, E.M. \& BACHA, C.J.C. A indústria brasileira de celulose de mercado e as pressões ambientais. Estudos Econômicos, 33 (1), 143-180, 2003.

HINOSTROZA, J.E.; \& MELLAR, H. Pedagogy embedded in educational software design: report of a case study. Computers \& Education, 37, 27-40, 2001.

HODSON, D. Time for action: science education for an alternative future. International Journal of Science Education, 25 (6), 645-670, 2003.

LITTLEJOHN, A.; FALCONER, I.; \& MCGILL, L. Characterising effective eLearning resources. Computers \& Education, 50, 757-771, 2008. 
MARJANOVIC, O. Using process-oriented, sequencing educational technologies: some important pedagogical issues. Computers in Human Behavior, 23, 2742-2759, 2007.

MARTÍNEZ ALIER, J. O ecologismo dos pobres. São Paulo: Contexto, 2007.

MARTÍNEZ PÉREZ, L. F.; PEÑAL, D. C.; \& VILLAMIL, Y. M. Relaciones ciência, tecnologia, sociedad y ambiente a partir de casos simulados: uma experiência em la enseñanza de la química. Ciência \& Ensino, 1 (Número especial), 2007. . Disponível em: <http://www.ige.unicamp.br/ojs/index.php/cienciaeensino/ >. Acessado em: 30/10/2008.

MORALES, R, LEEDER, D. and BOYLE, T. (2005) A case study in the design of generative learning objects (GLOs): applied statistical methods GLOs. Proceedings of ED-MEDIA 2005 (World Conference on Educational Multimedia, Hypermedia and Telecommunications). Montreal, Canada, June 27-July 2, 2005, pp 302-310. Disponível em: <http://www.ucel.ac.uk/glos/glo_info1.htm>. Acessado em: 16/07/2008.

NASCIMENTO, A.C.A.A. Objetos de aprendizagem: entre a promessa e a realidade. In: C. L. Prata \& A. C. A. A. Nascimento (Orgs.), Objetos de aprendizagem: uma proposta de recurso pedagógico (p. 135-146). Brasília: MEC, SEED, 2007.

OULTON, C.; DILLON, J. \& GRACE, M.C. Reconceptualising the teaching of controversial issues. International Journal of Science Education, 26 (4), 411423, 2004.

PARRAT-DAYAN, S. A discussão como ferramenta para o processo de socialização e para a construção do pensamento. Educação em Revista, 45, 13-23, 2007.

PARRISH, P.E. The trouble with learning objects. Educational Technology Research and Development, 52 (1), 49-67, 2004.

SCHIBECI, R.; LAKE, D.; PHILLIPS, R.; LOWE, K.; CUMMINGS, R. \& MILLER, E. Evaluating the use of learning objects in Australian and New Zealand schools. Computers \& Education, 50, 271-283, 2008.

SOLBES, J. \& VILCHES, A. STS interactions and the teaching of Physics and Chemistry. Science Education, 81, 377-386, 1997.

VACIK, H.; WOLFSLEHNER, B.; SPÖRK, J.; \& KORTSCHAK, E. The use of COCOON in teaching silviculture. Computers \& Education, 47, 245-259, 2006.

VAZ, L. Empresa de celulose destrói natureza para plantar eucalipto. Correio Braziliense, 01 de Junho de 2008. Disponível em: $<$ http://www.correiobraziliense.com.br>, contém vídeo. Acessado em: 30/10/2008, 2008a.

VAZ, L. O preço da devastação: reserva indígena degradada. Correio Braziliense, 02 de Junho de 2008. Disponível em: <http://www.correiobraziliense.com.br>, contém vídeo. Acessado em: 30/10/2008, 2008b.

VAZ, L. Empregados da Aracruz receberam área do governo e passaram para a empresa. Correio Braziliense, 03 de Junho de 2008. Disponível em: $<$ http://www.correiobraziliense.com.br>, contém vídeo. Acessado em: 30/10/2008, 2008c.

WHISNANT, D.M Scientific exploration with a microcomputer: simulations for nonscientists. Journal of Chemical Education, 61 (7), 627-629, 1984.

WHISNANT, D.M. A role-playing exercise using a computer simulation. Journal of Chemical Education, 69 (1), 42-43, 1992.

WILEY, D. A. Connecting Learning Objects to instructional design theory: a definition, a metaphor, and a taxonomy. In D. A. Wiley (Ed.), The instructional use of 
Learning

Objects,

2000.

Disponível

em:

<http://reusability.org/read/chapters/wiley.doc>. Acessado em: 20/08/2008. 